Please do not remove this page

RMIT

UNIVERSITY

\title{
Augmented band gap tunability in indium-doped zinc sulfide nanocrystals
}

Della Gaspera, Enrico; Griggs, Joseph; Ahmed, Taimur; Walia, Sumeet; Mayes, Edwin; Calzolari, Arrigo; Catellani, Alessandra

https://researchrepository.rmit.edu.au/esploro/outputs/9921863326401341/filesAndLinks?institution=61RMIT_INST\&index=null

Della Gaspera, E., Griggs, J., Ahmed, T., Walia, S., Mayes, E., Calzolari, A., Catellani, A., \& van Embden, J. (2019). Augmented band gap tunability in indium-doped zinc sulfide nanocrystals. Nanoscale, 11(7), 3360-3369. https://doi.org/10.1039/c8nr08830f

Document Version: Accepted Manuscript

Published Version: https://doi.org/10.1039/c8nr08830f

Repository homepage: https://researchrepository.rmit.edu.au

This journal is (c) The Royal Society of Chemistry 2019

Downloaded On 2023/04/26 18:20:46 +1000 


\section{Augmented Band Gap Tunability in Indium-}

\section{doped Zinc Sulfide Nanocrystals}

Enrico Della Gaspera ${ }^{a,}{ }^{,}$, Joseph Griggs ${ }^{a}$, Taimur Ahmed ${ }^{b}$, Sumeet Walia ${ }^{b}$, Edwin L. H. Mayes $^{c}$, Arrigo Calzolarid , Alessandra Catellanid, Joel van Embden ${ }^{a,{ }^{*}}$

a School of Science, RMIT University, Melbourne VIC 3000, Australia

b School of Engineering, RMIT University, Melbourne VIC 3000, Australia

c RMIT Microscopy and Microanalysis Facility, School of Science, RMIT University, Melbourne VIC 3000, Australia

d Istituto Nanoscienze CNR-NANO Centro S3, 41125 Modena, Italy

\section{Corresponding Authors}

*Email: enrico.dellagaspera@rmit.edu.au (EDG); joel.vanembden@rmit.edu.au (JvE)

\section{Keywords}

band gap, quantum confinement, ZnS, colloids, doping, UV sensor 


\begin{abstract}
Doping semiconductor nanocrystals is a powerful tool to impart new and beneficial optical and electrical properties to the host nanocrystals. Doping has been used to improve the performances of nanocrystals-based devices in applications as diverse as optics, magnetism, electronics, catalysis and sensing. In this work we present a low temperature colloidal synthesis of zinc sulfide $(\mathrm{ZnS})$ nanocrystals doped with indium. Through optimization of the reaction parameters and the doping level, quantum confined ( $\sim 2 \mathrm{~nm}$ in size) crystalline colloids with highly tunable optical properties are achieved. Using a suite of characterization techniques including x-ray diffraction, high-resolution transmission electron microscopy, optical spectroscopies (absorption, emission, and Raman), compositional analyses and first principles simulations, we investigate the structural, morphological and optical properties of the synthesized nanocrystals. Indium dopants are found to heavily influence the band gap of ZnS. This strategy in addition to traditional methods of size control enables the synthesis of nanocrystals (with finely tunable band gaps between $\sim 3.8 \mathrm{eV}-4.3 \mathrm{eV}$ ). These doped $\mathrm{ZnS}$ nanocrystals are fabricated into selective UV thin-film absorbers and discriminatory proof-of-concept UVA-UVB/C photo-detectors.
\end{abstract}




\section{Introduction}

Research on colloidal semiconducting nanocrystals (NCs) has been constantly increasing since the seminal work conducted in the 1980s and 1990s, when fundamental properties such as the quantum confinement effect were hypothesized and demonstrated.1,2 Over the course of many years the nanomaterial research community has acquired a deep understanding of the distinctive phenomena displayed by colloidal NCs. To date, a multitude of synthetic protocols has been developed to synthesize a wide array of nanocrystals and quantum-confined colloids. $3,4,5,6$ This has enabled the use of these nanomaterials in a large variety of applications, including optics and optoelectronics (light emitting diodes, photodetectors, lasers, solar cells smart windows), catalysis, sensing and energy storage. ${ }^{7,8,9,10,11}$

Zinc sulfide $(\mathrm{ZnS})$ is a well-studied semiconductor due its large direct band gap, high refractive index, and excellent stability towards oxidation and hydrolysis. The amenability of ZnS NCs to the incorporation of dopants ( $p$ - and $n$-type) can be used to further impart enhanced properties to these NCs (and indeed other nanoscale materials in general). As such, doped ZnS nanomaterials have shown both improved luminescence and conductivity. ${ }^{12,13,14,15}$ In addition, $d$-doping of ZnS NCs has been reported as a means to enhance visible luminescence. ${ }^{16}$ Collectively, these inherent properties and their modifications have enabled the use of $\mathrm{ZnS}$ in a wide range of applications, such as UVblocking and visible-transparent coatings, shelling material for core-shell quantum dots, luminescent nanocomposites, transistors, catalysts and chemical sensors. ${ }^{12,13,17,18,19}$

Zinc sulfide NCs have been synthesized through various established techniques, including high temperature colloidal syntheses and co-precipitation methods. ${ }^{13,20,21}$ High temperature methods in non-polar solvents are time consuming because they typically require an extensive washing procedure to remove the by-products, especially sulfur in the form of a sulfur-oleylamine complex. ${ }^{22}$ Conversely, ZnS NCs synthesized through coprecipitation methods typically lack colloidal stability and show poor control over particle size and distribution. While additional methods for colloidal syntheses at low temperature using polar solvents such as alcohols have been demonstrated, they still lack the ability to effectively tune the ZnS NC size and control polydispersity. Consequently, they do not provide fine control over size-dependent properties, such as band gap and photoluminescence (PL). ${ }^{23,24,25}$ 
In this work, we simultaneously address all these issues by presenting a novel reaction for the low temperature synthesis of quantum confined pure and doped $\mathrm{ZnS}$ colloids. The physical and optical properties of the NCs were examined using a suite of experimental characterization techniques as well as through ab initio calculations. The methods outlined herein utilize low reaction temperatures $\left(<130^{\circ} \mathrm{C}\right)$ and produce highly crystalline NCs with tunable absorption (band gap) and emission (PL) properties. In addition, utilizing these optimized reaction protocols ZnS NCs may be aliovalently doped with indium with near perfect control over dopant level incorporation, i.e. near quantitative conversion of dopants from the reaction mixture into the host $\mathrm{ZnS} \mathrm{NC}$ matrix. Notably, the incorporation of indium dopants provides an additional means to finely control the band gap through relaxation of quantum size effects. The optimized doped ZnS NCs developed here can be readily processed into colloidal "inks" for use in the fabrication of thin-film coatings. Using the synthetic methods outlined herein indium doped ZnS NCs were fabricated into proof-of-concept UV sensors capable to selectively detect specific subclasses of UV radiation (UVB/C over UVA).

\section{Results and Discussion}

We synthesized ZnS-based NCs through the reaction of zinc acetate and indium acetylacetonate with thioacetamide in a coordinating solvent $(\mathrm{N}, \mathrm{N}$-dimethyl formamide, DMF) using 5-amino-1-pentanol to impart additional colloidal stability to the final NCs. Complete details are reported in the Experimental section. After the exploration of multiple prospective solvents, it was found that DMF was the most suitable due to a series of advantages including (i) its ability to effectively dissolve commonly used metal precursors such as acetates and acetylacetonates; (ii) its ability to coordinate metal ions; (iii) its mildly reducing nature which provides a suitable reaction environment avoiding oxide formation; (iv) its boiling point $\left(153^{\circ} \mathrm{C}\right)$, which enables the reaction to be conducted at elevated reaction temperatures compared to established chemical syntheses in polar solvents (such as alcohols). ${ }^{25} \mathrm{ZnS}$ NCs were synthesized at various temperatures $\left(60^{\circ} \mathrm{C}, 90^{\circ} \mathrm{C}\right.$ and $130^{\circ} \mathrm{C}$ ) and with various indium dopant levels (up to $20 \%$ molar doping with respect to zinc). The as-prepared colloids were easily purified by means of simple centrifugation and re-dispersion cycles (see Experimental). 

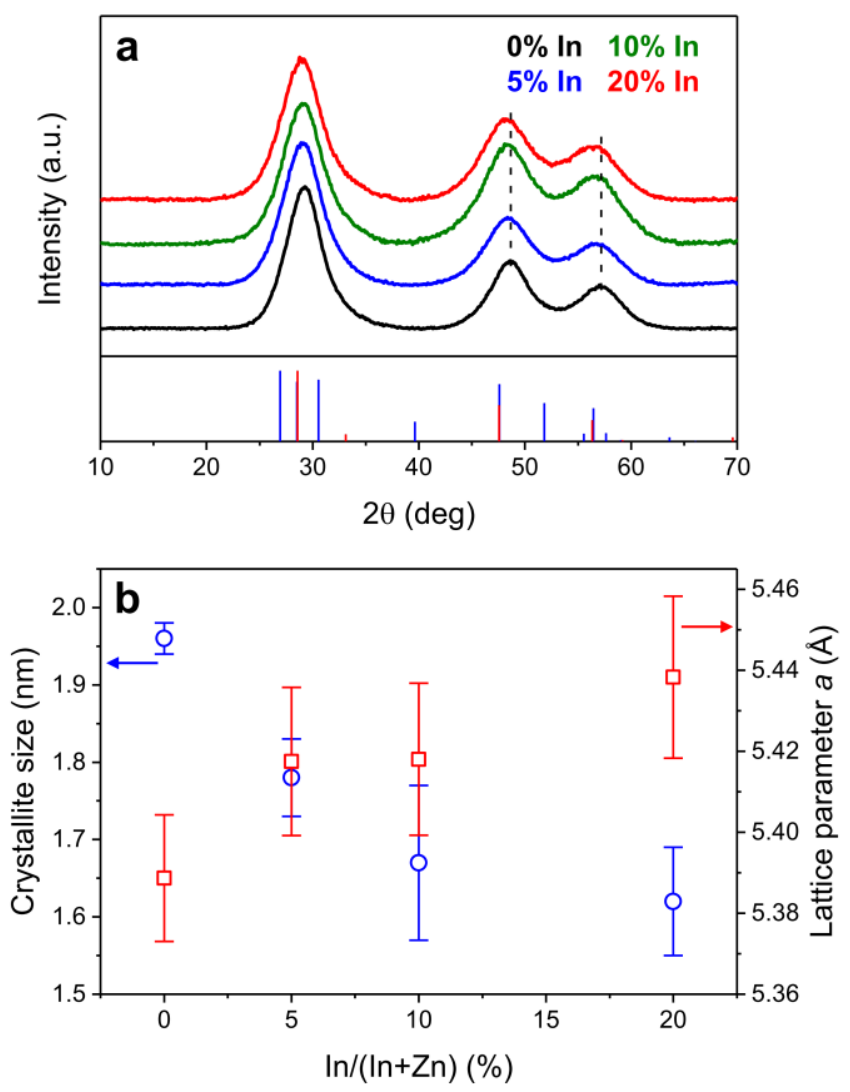

Figure 1. a) X-ray diffraction patterns of the samples synthesized at $130{ }^{\circ} \mathrm{C}$ with different indium doping levels. The predicted diffraction peak positions for cubic (red) and hexagonal (blue) ZnS are displayed at the bottom. The vertical dashed lines mark the peak positions for the undoped sample, highlighting the peak shift with increasing indium doping. b) Crystallite size and lattice parameter calculated from the diffraction patterns presented in (a).

Figure 1a shows the X-ray diffraction patterns for ZnS samples synthesized at 130 ${ }^{\circ} \mathrm{C}$ with different indium dopant concentrations. All patterns can be indexed to cubic sphalerite (zincblende) ZnS (ICDD No. 05-0566). The broadening of the diffraction peaks is consistent with the domain size of the prepared colloids being on the nanometer scale. It is well-known that $\mathrm{ZnS}$ can also crystallize in a hexagonal wurtzite phase (ICDD No. 361450). When the crystallite size is just a few nanometers, the convolution of broad diffraction peaks makes it more difficult to distinguish between these two phases using XRD. Nevertheless, the absence of any notable signal from the (102) wurtzite diffraction peak at $\sim 39.6^{\circ}$ provides strong evidence that the as-prepared nanocrystals adopt a purely cubic structure. Importantly, no impurity peaks arising from zinc oxide or indiumrich phases were observed in the XRD patterns of the prepared NCs, with the single exception of samples synthesized at very low temperatures $\left(60^{\circ} \mathrm{C}\right)$ with the highest level 
of indium doping (20\%, see Figure S1 in ESI). The impurity in this sample is likely to be indium oxide or indium sulfide. This highlights that higher reaction temperatures facilitate indium incorporation into the $\mathrm{ZnS}$ host lattice, while very low reaction temperatures favor the formation of homogeneously nucleated indium-based impurities.

Close analysis of the XRD diffraction patterns in Figure 1a reveals an increase in peak broadening and a slight shift of the peaks towards smaller angles at increased indium doping. Peak broadening may be used to estimate the crystallite size through the Scherrer formula, wherein smaller crystallite sizes display increased broadening. Results from Scherrer analyses after fitting the diffraction peaks with Pseudo-Voigt functions (see Figure S2 in ESI for details on the peak fitting) are shown in Figure 1b, which reveals a progressive decrease in crystallite size with increasing indium doping for all prepared samples. Specifically, a decrease in crystallite size from $1.96 \pm 0.02 \mathrm{~nm}$ to $1.62 \pm 0.07 \mathrm{~nm}$ is calculated for NCs doped with the largest amount of indium compared to undoped NCs (corresponding to a $\sim 50 \%$ reduction in NC volume). Furthermore, this relative decrease in size (volume) was found to be irrespective of the reaction temperature (Table S1 in ESI). As such, it is clear that indium dopants strongly perturb the nucleation and growth processes of ZnS NCs.

The peak shift is consistent with a slight expansion of the ZnS lattice caused by the larger trivalent indium ions compared to divalent zinc ions. Calculation of the lattice parameter, $a$, for a cubic cell using the position of the diffraction peaks and the respective Miller indices shows a clear trend in cell expansion with indium doping (Figure 1b). The cell expansion is ascribed to indium-induced stresses within the $\mathrm{ZnS}$ host lattice. This is confirmed by first principles simulations performed on $\mathrm{ZnS}$ single cubic crystals, showing an almost linear increase in the unit cell of $\mathrm{ZnS}$ with the addition of substitutional indium dopants (Figure S3 in ESI). To analyze the amount of indium that was effectively incorporated within the $\mathrm{ZnS}$ lattice, atomic emission spectroscopy was employed after dissolving the highly purified NCs in concentrated acid. The presence of indium was readily confirmed in all doped samples (see Table 1), with its concentration matching excellently to the nominal doping amounts. Notably, in some cases the amount of indium is slightly larger than expected. This can be explained by the higher reactivity of indium acetylacetonate with respect to zinc acetate, and given fact that not all zinc precursors reacted (reaction yield lower than unity). 


\begin{tabular}{cccc}
\hline $\begin{array}{c}\text { Reaction } \\
\text { temperature }\left({ }^{\circ} \boldsymbol{C}\right)\end{array}$ & $\begin{array}{c}\text { Nominal } \\
\text { In/(Zn+In) } \%\end{array}$ & $\begin{array}{c}\text { Experimental } \\
\text { In } /(\mathbf{Z n}+\mathbf{I n}) \%\end{array}$ & $\begin{array}{c}\text { Variation } \\
\text { In } /(\mathbf{Z n}+\mathbf{I n}) \%\end{array}$ \\
\hline 130 & 0 & 0.02 & +0.02 \\
130 & 5 & 6.6 & +1.6 \\
130 & 10 & 12.4 & +2.4 \\
130 & 20 & 18.7 & -1.3 \\
90 & 5 & 7.3 & +2.3 \\
90 & 10 & 12.5 & +2.5 \\
60 & 5 & 6.6 & +1.6 \\
60 & 10 & 10.2 & +0.2 \\
\hline
\end{tabular}

Table 1. Comparison of nominal (amount of precursor used) and experimental (from AES) indium doping levels as a function of the reaction temperature for the key samples synthesized in this study.

Transmission electron microscopy (TEM) was also used to analyze the prepared colloids. Figure 2 shows both low-resolution (Figure $2 \mathrm{a}$-d) and high resolution (e-h) TEM images of nanocrystals synthesized at $130{ }^{\circ} \mathrm{C}$ with various indium doping levels. All NCs are within the 2-3 nm size range, with a slight reduction in size for highly doped NCs (as anticipated by XRD analyses). Importantly, they are all highly crystalline despite their small size, as confirmed by observation of their strong lattice fringes, and the respective Fast Fourier Transform (FFT) images reported as insets. Additional TEM data and FFT images are reported in Figure S4 in ESI. Selected area electron diffraction patterns of representative samples are also presented (Figure $2 \mathrm{i}, \mathrm{j}$ ). All observed $d$-spacings match well to those of cubic $\mathrm{ZnS}$, and no reciprocal lattice spacings from the wurtzite phase could be detected. Furthermore, statistical analysis of FFT images obtained from high resolution images of the NCs with different amount of indium doping confirmed once more that indium incorporation results in a significant lattice expansion (Figure 2k). In detail, the $d$-spacing associated with the (111) plane has been measured at $3.08 \pm 0.04 \AA$ for undoped ZnS, $3.13 \pm 0.05 \AA ̊$ for $5 \%$ indium doping, $3.15 \pm 0.04 \AA$ for $10 \%$ doping and $3.23 \pm 0.04 \AA$ for $20 \%$ doping. This trend is almost linear (see Figure S5 in ESI) and matches very well the prediction of our model, as shown in Figure S3 in ESI. 


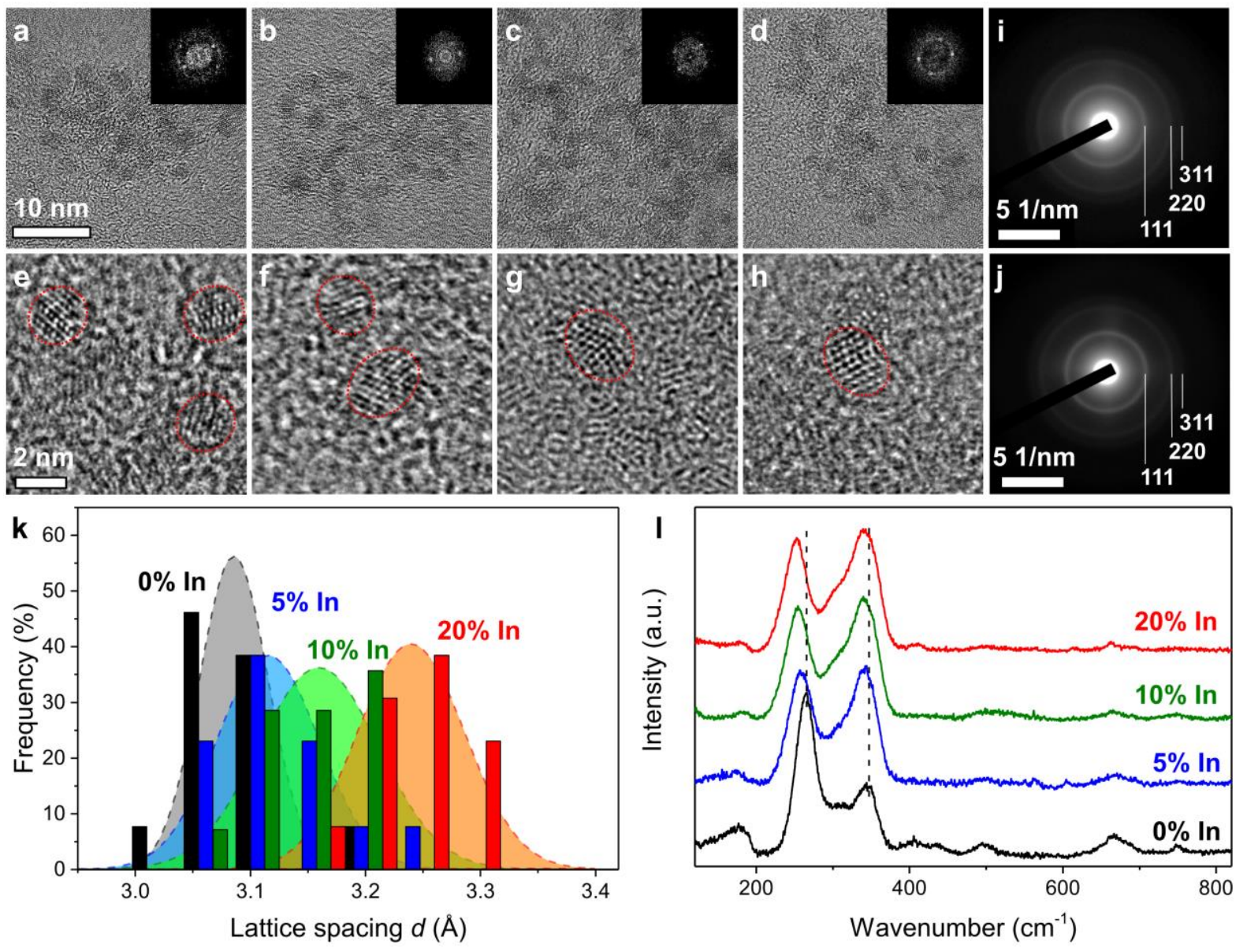

Figure 2. Low resolution TEM (a-d) and high resolution TEM (e-h) images for undoped $(\mathrm{a}, \mathrm{e}), 5 \%(\mathrm{~b}, \mathrm{f}), 10 \%(\mathrm{c}, \mathrm{g})$ and $20 \%(\mathrm{~d}, \mathrm{~h})$ indium doped samples synthesized at $130{ }^{\circ} \mathrm{C}$. The insets in the upper panels show the Fast Fourier Transform (FFT) images. The electron diffraction patterns for undoped (i) and 10\% indium doped (j) samples are also reported. k) Histogram showing the statistics of the $d$ spacing corresponding to the (111) lattice plane measured from several FFT images (see also Figure S4 in ESI). l) Raman spectra of samples synthesized at $130{ }^{\circ} \mathrm{C}$ with different indium doping levels.

Raman spectroscopy (Figure 2l) also revealed some differences in the prepared ZnS NCs as a function of indium doping. All samples showed prominent vibrational peaks in the 200-400 $\mathrm{cm}^{-1}$ range, and some additional lower intensity peaks below $200 \mathrm{~cm}^{-1}$ as well as between 400 and $800 \mathrm{~cm}^{-1}$. The main peaks can be ascribed to the first order transverse optical (TO) and longitudinal optical (LO) phonon modes of cubic $\mathrm{ZnS}$ centered at $\sim 260 \mathrm{~cm}^{-1}$ and $\sim 350 \mathrm{~cm}^{-1}$, respectively. ${ }^{26}$ These peaks are seen to concomitantly shift to lower wavenumbers with increasing indium doping as a consequence of introducing strain into the lattice (from larger indium atoms) similar to the effects observed from the XRD results. ${ }^{27}$ Interestingly, these peaks are also seen to 
broaden slightly with increasing indium doping. This effect is due to the different crystallite size, as observed previously for other nanocrystalline materials including $\mathrm{TiO}_{2}$ and silicon, and is consistent with the slightly smaller size of the doped ZnS NCs with respect to their undoped counterparts. ${ }^{28,29}$ The other weak Raman peaks are ascribed to second order phonon modes with their intensity and position being consistent with previous literature reports. ${ }^{30,31}$ No additional peaks were detected in the indium doped samples, further confirming the absence of impurity phases.

In addition to their clear effects on the crystal structure of ZnS NCs, indium dopants are also responsible for modifications to their optical properties. Figure 3 shows the optical properties of the prepared ZnS NCs as a function of reaction temperature and doping levels. A sharp UV absorption onset is observed for all ZnS NC solutions, consistent with the large direct band gap of zinc sulfide. The observed band gap energies of the NCs are greater than bulk cubic $\mathrm{ZnS}(\sim 3.7 \mathrm{eV})$ as a consequence of the quantum confinement effect. ${ }^{17,32}$ Increasing the reaction temperature from $60{ }^{\circ} \mathrm{C}$ to $130{ }^{\circ} \mathrm{C}$ results in a concomitant red shift of the band gap (Figure 3a) indicating that the size of the NCs increases systematically with growth temperature. This is consistent with the crystallite sizes obtained from both XRD and Raman analyses discussed earlier. The PL spectra are also seen to red shift with increasing reaction temperature. It is important to note here that the observed PL emission is predominantly due to surface defects rather than bandto-band recombination, as evident from the broad and highly red-shifted PL peaks with respect to the band edge. The PL peak corresponding to direct electron relaxation from conduction band to valence band is barely visible and is observed only as a small shoulder in the PL peaks at slightly lower energies than the band gap energy (see also Figure S6 in SI). The predominance of emission from defect states (over band gap emission) is very common for ZnS quantum dots, as well as for other analogous II-VI semiconductor colloids such as CdS and ZnO. 20,33,34 

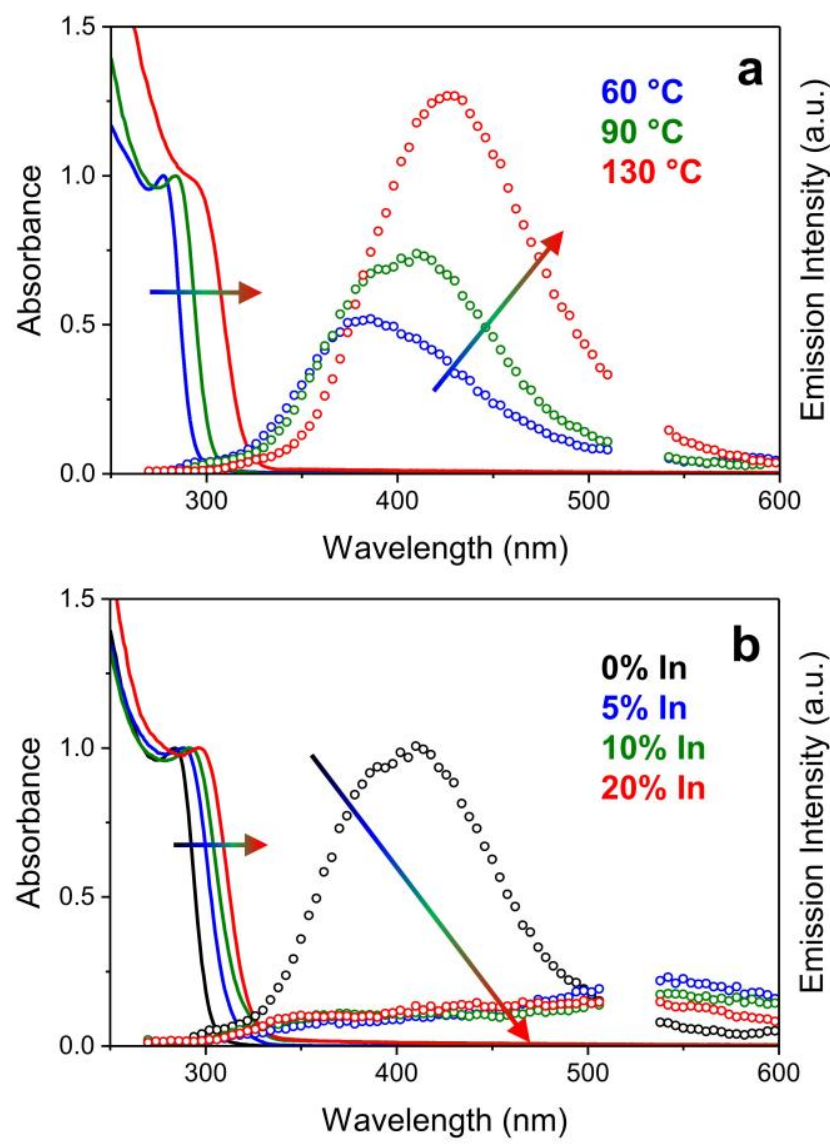

Figure 3. Combined absorption (straight lines) and emission (open circles) spectra (excitation at $260 \mathrm{~nm}$ ) for a) undoped ZnS NCs synthesized at different temperatures and b) for ZnS NCs synthesized $90{ }^{\circ} \mathrm{C}$ with different indium doping levels. All spectra were obtained from NCs dispersed in chloroform.

Figure $3 \mathrm{~b}$ shows the absorption and PL spectra for $\mathrm{ZnS}$ synthesized at $130{ }^{\circ} \mathrm{C}$ with various concentrations of indium dopant. It can be seen that indium doping dramatically decreases the PL emission from sub band gap states. This observation is a consequence of either (i) a decrease in surface defects or (ii) the further addition of energy levels within the band gap, which either result in the emission of low-energy (infrared) photons or provide non-radiative (phonon assisted) pathways for electron decay. It is important to note here that a reduction in surface defects for quantum dot colloids is invariably associated with an increase in band gap PL (which was not observed here). As such we attribute quenching of the "trap" PL band to the dopant induced generation of multiple sub-band gap energy levels and non-radiative decay pathways. A similar effect has been reported in various aliovalently doped semiconductors, including In-doped $\mathrm{ZnO}$ nanofibers, Ag-doped InAs NCs and B-doped Si NCs. ${ }^{35,36,37}$ Importantly, increasing the concentration of indium also culminates in a red-shift of the band gap of the host ZnS NCs. 
This provides an additional parameter other than reaction temperature, which can be used to modulate the absorption onset of $\mathrm{ZnS}$.

It is clear that indium doping has effects on both the growth kinetics and the band gap of ZnS NCs. As discussed earlier, doped ZnS NCs are slightly smaller in size compared to their undoped counterparts. As such, from quantum confinement theory we expect to observe a blue-shift of the absorption onset upon doping, which is contrary to what is observed. Consequently, given the observation of a concomitant red-shift of the absorption spectrum with indium doping, the primary effect of indium dopants must be to decrease the band gap. This highlights the strong effect that indium exerts on the electronic structure and band gap of $\mathrm{ZnS}$, as despite the competing effects of quantum confinement, the net effect of doping is a red-shift of the absorption onset.

To further clarify this key aspect, in Figure 4 we summarize the relationship between the band gap energy and the crystallite size as a function of indium doping for ZnS synthesized at different temperatures. A combined plot mapping the band gap energy as a function of size and indium doping is presented in Figure S7 in ESI. Analysis of Figure 4 shows that for any given indium doping level, higher reaction temperatures result in an increase in NC size and a decrease in band gap energy as expected according to quantum confinement theory. In contrast, for a given reaction temperature, higher doping levels cause a concurrent decrease in size and a decrease in band gap energy. Therefore, we have demonstrated the specific role of indium dopants in reducing the band gap of ZnS regardless of the NCs size. Given that the bulk band gap of indium sulfide ranges between 2.1 and $2.8 \mathrm{eV}$, it is clear that indium doping relaxes the quantum size effects prevalent in the host $\mathrm{ZnS} \mathrm{NC}$, similarly to what has been observed for other alloyed semiconductors. ${ }^{38,39,40}$ The Tauc plot used to estimate the band gap for several key $\mathrm{ZnS}$ based samples is presented in Figure 4c. The band gaps are seen to be highly direct regardless of dopant concentration or synthesis temperature. Through the dual effect of reaction temperature and indium doping, the band gap can be precisely adjusted across a wide energy range (3.8 eV-4.3 eV). Notably, this energy range spans the UVA/UVB range and therefore the reaction conditions can be easily adjusted to selectively absorb these regions of the UV spectrum. 

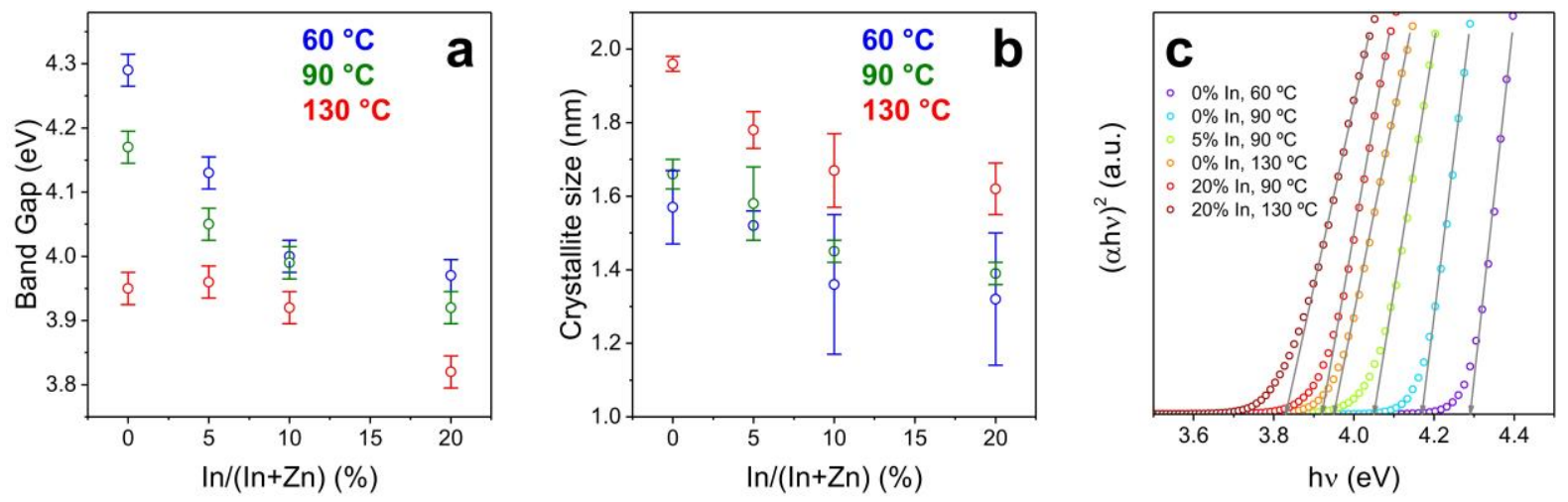

Figure 4. a) Band gap energy and b) crystallite size as a function indium doping for ZnS NCs synthesized at different temperatures. Both band gap energy and NC size decrease with indium doping. c) Tauc plot assuming direct transition for representative samples showing band gap tuneability from $\sim 3.8 \mathrm{eV}$ to $\sim 4.3 \mathrm{eV}$.

To further investigate this unusual modulation of the optical band gap of zinc sulfide due to aliovalent indium doping, we performed $a b$ initio simulations of the optoelectronic properties of zincblende $\mathrm{ZnS}$, at various In doping levels (see Experimental for details). We discuss the modifications induced by indium doping, by comparing the Density of States (DOS) obtained at different indium content, namely 3, 10 and $20 \%$ (corresponding to the substitution of $1 / 32,3 / 32$ and $6 / 32 \mathrm{Zn}$ atoms, in a 64 atom supercell), as shown in Figure 5 and Figure S8 in ESI. In contrast with what happens in $\mathrm{ZnO}$ (see also Figure S9 in ESI), when inside ZnS lattice indium does not donate its 5p electron to the host, but rather inserts occupied defect states in the gap (in-gap states IGSs): this reduces the optical gap and also the electron conductivity. The effect of these IGSs is clearly revealed by comparing the optical response of undoped and doped ZnS. In Figure S10 in ESI we show the imaginary component of the dielectric function $\left(\varepsilon_{2}\right)$ for undoped and $10 \%$ doped $\mathrm{ZnS}$ : while $\varepsilon_{2}$ has the expected onset at the energy gap threshold for the pure host, the doped system presents an irregular absorption and degradation of optoelectronic properties at lower energies. This shows that indium-doped ZnS does not behave as transparent conductor, nor a plasmonic material (Figure S11 in ESI), since no or very small free electronic charge is donated by the dopant to the host. This point can be easily clarified through the analysis of the ionization energies of the different compounds. The ionization potential of indium is too low with respect to the electron affinity of $\mathrm{ZnS}$, laying almost mid-gap, at variance with $\mathrm{ZnO}$ where the EA is instead comparable to indium ionization energy (see Figure S12 in ESI). Therefore, indium acts 
as a deep donor within $\mathrm{ZnS}$ and is not capable to provide free charge in the $\mathrm{ZnS}$ conduction band, but is responsible for IGSs formation at $\sim 1 \mathrm{eV}$ below the pristine conduction band bottom of the $\mathrm{ZnS}$ host. This is in stark contrast with $\mathrm{ZnO}$, where indium act as a shallow donor, providing free electrons within the conduction band of $\mathrm{ZnO}$, a foundation for the use of In-doped $\mathrm{ZnO}$ as a transparent conductor and an infrared plasmonic material. 41,42

Furthermore, in-gap states associated to indium dopant also appear in $\mathrm{ZnS}$ doped NCs, where indium is preferentially located at the NC surface, causing a band gap reduction compared to undoped ZnS NCs (see Figure S13 in ESI).

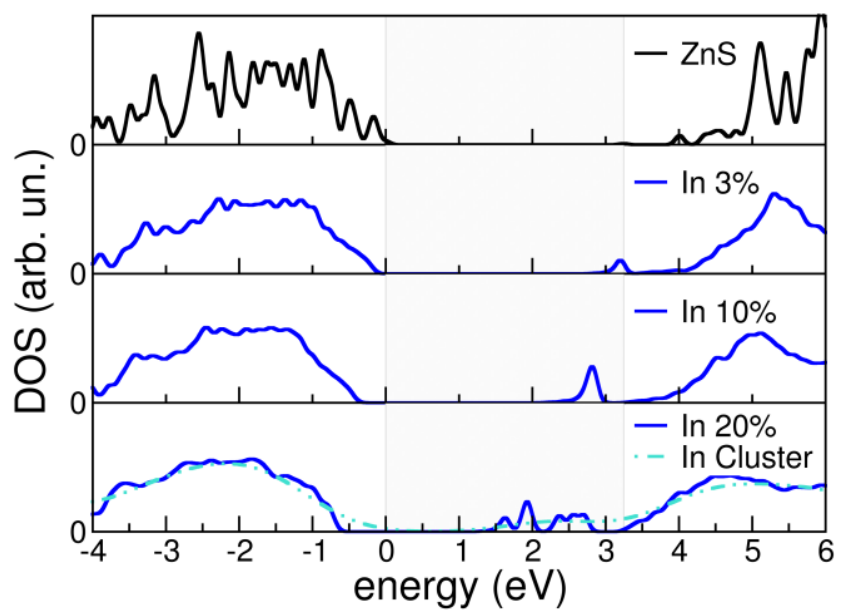

Figure 5. DOS of In:ZnS bulk systems at different In contents. The top of the valence band of the undoped $\mathrm{ZnS}$ host bulk was considered as energy reference $(\mathrm{E}=0 \mathrm{eV})$; a grey box represents the pristine band gap of the host. Results for regularly distributed In atoms are compared with those obtained for aggregates of substitutional In, here named "Cluster", to evaluate the effect of possible $\operatorname{In}_{2} \mathrm{~S}_{3}$ inclusions.

The as-prepared colloids can be easily purified and transferred into various solvents using conventional ligand exchange procedures to obtain stable suspensions in either polar (e.g. propanol, ethanol) or non-polar (e.g. chloroform, hexane) solvents. The surface ligands (5-amino-1-pentanol for polar solvents and oleylamine for non-polar solvents) impart excellent colloidal stability to the solutions and enable concentrated (40-50 mg/mL) nanoparticle "inks" to be formulated, which are suitable for thin film deposition. ${ }^{43,44}$ The ability to readily process these NCs into thin-film coatings is a key step for the development of high-performance devices such as solar cells, LEDs, transistors, printed electronics in general and various optical sensors. $44,45,46,47,48$ High quality, smooth thin films could be deposited on various substrates including glass and silicon. Figure S14 shows the refractive index and the extinction coefficient evaluated 
using ellipsometry for thin $\mathrm{ZnS}$ films deposited on silicon. The refractive index values in the visible spectrum for these films range between 1.7 and 1.8 regardless of indium doping. These are much lower values compared to the expected refractive index for bulk zinc sulfide $(\sim 2.37$ at $589 \mathrm{~nm}) .{ }^{49}$ This may be ascribed to the inherent porosity of the thin films, which are composed of a solid particle network and void spaces. Evaluation of the porosity of these coatings using the Bruggemann formula suggests porosity values around $40 \%$ for all samples prepared. 50
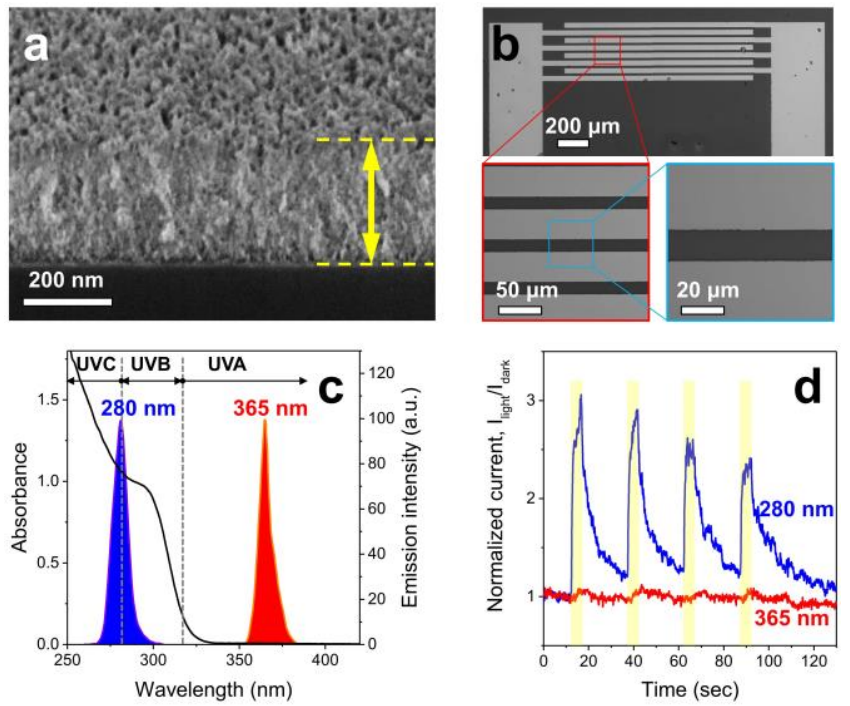

Figure 6. a) SEM image in cross section at 55 degrees tilt of a ZnS:In sample (10\% doping). The double-headed arrow in identifies the film thickness. b) Digital photographs at different magnifications of a $\mathrm{ZnS}$ film deposited on a $\mathrm{Si} / \mathrm{SiO}_{2}$ substrate which was prepatterned with inter-digitated gold electrodes. c) Emission spectra of the two LEDs used for photoconductivity measurements and comparison with the absorption spectrum of the ZnS:In colloids (10\% doping). d) Normalized current as a function of time for a ZnS film deposited on pre-patterned electrodes and exposed to pulsed $280 \mathrm{~nm}(\mathrm{UVB} / \mathrm{C})$ and a $365 \mathrm{~nm}$ (UVA) LED. The yellow shaded areas identify the time period the samples were illuminated.

Figure 6a shows a scanning electron microscopy (SEM) image (in cross section) of a typical ZnS NC thin film highlighting the uniform surface and homogeneous thickness (for top view SEM see Figure S15 in ESI). The porosity of the film can also be clearly seen, corroborating the refractive index results. SEM was also used to analyze sample composition through energy dispersive x-ray (EDX) measurements. The results from EDX substantiate those obtained from AES (Table S2 in SI), showing indium dopant levels consistent with their nominal amounts. 
ZnS nanocrystals and thin films can be used in several applications, including gas and UV sensors, light emitting diodes, buffer layers for solar cells, UV-blocking coatings or as a host matrix for luminescent quantum dots. ${ }^{17,25,51,52}$ As an example, in this work we utilize our doped ZnS NCs as selective UV sensors, which can discriminate UVA from $\mathrm{UVB} / \mathrm{C}$ radiation. Similar to the more studied $\mathrm{ZnO}$, the band gap of bulk $\mathrm{ZnS}$ lies in the UVA range (3.1-3.95 eV), and as such both $\mathrm{ZnS}$ and $\mathrm{ZnO}$ have been used as a UV sensors which are also insensitive to visible radiation. ${ }^{17,53,54}$ However, ZnS has a wider band gap with respect to $\mathrm{ZnO}$, which makes it amenable to deep $\mathrm{UV}$ applications that require distinction between higher energy UVB/C and lower energy UVA radiation. ${ }^{55}$ Conversely, the ability to precisely tune the band gap of our ZnS NCs through both quantum confinement and doping allows the fabrication of selective UVB/C sensors that are unaffected by UVA or visible radiation. Figure $6 \mathrm{~b}$ shows an optical micrograph of the sensor design fabricated using lithographic processes (see Experimental for fabrication details). To obtain the working sensor a thin film of ZnS NCs is deposited on the prepatterned electrodes and exposed to either UVA or UVB/C light, through the use of a 365 $\mathrm{nm}(3.4 \mathrm{eV})$ and a $280 \mathrm{~nm}(4.4 \mathrm{eV}) \mathrm{LED}$, respectively (Figure 6c). As can be seen from Figure 6d, a distinct and reversible photocurrent is detected when the material is exposed to higher energy UVB/C (280 nm). However, negligible photocurrent is observed for UVA exposure. This result validates the use of ZnS NCs for selective UV radiation detection. Moreover, by either depositing thin layers of NCs, or by embedding ZnS in a transparent host matrix, visibly transparent coatings with selective UV absorption properties can be fabricated (Figure S16 in ESI), which could find application as optical filters and absorbers of harmful UVB/C radiation.

\section{Conclusion}

In conclusion, we presented the synthesis of colloidal ZnS NCs doped with indium with highly selective absorption properties in the UV range. We achieved near perfect control over the amount of indium inclusion to levels up to $20 \%$ without the segregation of indium impurities. Through the investigation of the optimized pure and doped $\mathrm{ZnS}$ nanocrystals, we identified the role of alloying indium dopants on the optical and structural properties of ZnS. Specifically, we demonstrate that indium incorporation can be used to predictably red shift the optical absorption onset. Notably, this effect was 
found to be independent of NC size. Using the methods outlined herein the absorption onset can be fine-tuned over a wide energy range (3.8-4.3 eV), which enables the band gap to be tuned to discriminate between UVA and UVB radiation. This work represents a marked advancement in the synthesis of $\mathrm{ZnS}$-based materials, and it is our hope that the findings outlined here leverage future studies into the effects of a wider range of dopants for $\mathrm{ZnS}$.

\section{Experimental}

\section{Materials}

Zinc acetate dihydrate (99\%), indium (III) acetylacetonate (99.99\%), N,Ndimethyl formamide (DMF, 99.9\%), 5-Amino-1-Pentanol (AP, 95\%), thioacetamide (99\%), oleylamine (OLA, 70\%), ammonium sulfide (40-48\% in water) and chloroform (99.5\%) were supplied by Sigma-Aldrich. Acetone (99\%) was purchased from Chem Supply. $n$-hexane (95\%) was supplied by Univar. All chemicals were used without further purification.

\section{ZnS NCs synthesis}

ZnS NCs were synthesized by reacting metal carboxylate precursors with thioacetamide in DMF at different temperatures in the presence of AP as surface ligand. In all reactions the sum of the moles of zinc and indium precursors was kept constant. The molar indium content was changed between 0 and $20 \%$ with respect to zinc according to the following:

$$
\operatorname{In}(\%)=\frac{n_{I n}}{\left(n_{Z n}+n_{I n}\right)} \times 100
$$

where $n$ is the number of moles.

In a typical reaction, $2 \mathrm{mmol}$ of zinc and indium precursors in the desired ratio were dissolved in $27 \mathrm{~mL}$ of DMF in the presence of $2.6 \mathrm{mmol}(268.2 \mathrm{mg}) \mathrm{AP}$ in a $100 \mathrm{~mL}$ 3-neck round bottom flask. As an example, for a 10\% indium doping, $1.8 \mathrm{mmol}$ of zinc acetate dihydrate $(395.1 \mathrm{mg}$ ) and $0.2 \mathrm{mmol}$ of indium acetylacetonate $(82.4 \mathrm{mg}$ ) were used. The flask was connected to a Schlenk line and degassed briefly ( $\sim 1$ minute) under vacuum at room temperature, and then purged with nitrogen. This short degas procedure was repeated once more to ensure most of oxygen was eliminated from the reaction environment while preventing excess evaporation of DMF. The temperature was then 
increased to the desired value $\left(60^{\circ} \mathrm{C}, 90^{\circ} \mathrm{C}\right.$ or $\left.130{ }^{\circ} \mathrm{C}\right)$. When the desired temperature was reached, a freshly made solution of thioacetamide (2 mmol, $105.3 \mathrm{mg}$ ) in 3mL DMF was rapidly injected into the precursor solution under strong stirring. The reaction was continued for one hour (at the injection temperature), and then the flask was removed from the heater and cooled to room temperature with the aid of a water bath.

\section{ZnS NC purification and thin-film deposition}

The as-synthesized NCs could then be purified through centrifugation and redispersion protocols, and transferred into different solvents using conventional ligand exchange procedures. Specifically, the ZnS NCs can be precipitated from DMF using a 1:1 mixture of acetone and $n$-hexane, centrifuged at $4400 \mathrm{rpm}$ for 5 minutes and resuspended in 1-propanol with the aid of additional AP to afford colloidal stability. This procedure could be repeated to further purify the NCs. To obtain dry powders (for XRD), no additional ligand was used.

The NCs were transferred to non-polar solvents, replacing polar AP with bulkier OLA ligands. The procedure is as follows: $15 \mathrm{~mL}$ of the as-synthesized NCs in the mother liquor was mixed with $20 \mathrm{~mL}$ ethanol, $15 \mathrm{~mL}$ acetone and $2 \mathrm{~mL}$ OLA, causing instant flocculation of the NCs. After centrifugation (4400 rpm, $5 \mathrm{~min}$ ) the supernatant was discarded and the precipitate was re-suspended in chloroform with excess OLA. Multiple washing of the NCs could be carried out using ethanol as non-solvent and chloroform (or other non-polar solvents such as toluene and octane) as solvent, with the care of adding additional OLA between washes to improve the colloidal stability of the NCs and drive the desorption of residual AP molecules.

Thin films were deposited using spin coating from concentrated $(\sim 30 \mathrm{mg} / \mathrm{mL}) \mathrm{NC}$ suspensions in 1-propanol at $2000 \mathrm{rpm}$ for 30 seconds (60-80 nm per layer). The films were then annealed at $180{ }^{\circ} \mathrm{C}$ for 2 minutes in air. For the electrical measurements, multilayer films were fabricated. After each ZnS NC layer was annealed at $180^{\circ} \mathrm{C}$, the film was dipped in a solution of ammonium sulfide (prepared mixing $300 \mu \mathrm{L}$ of a $40-48 \%$ $\left(\mathrm{NH}_{4}\right)_{2} \mathrm{~S}$ solution in water with $10 \mathrm{~mL}$ of ethanol) for 5 minutes to remove the AP ligands, then re-annealed at $180^{\circ} \mathrm{C}$. This procedure enables to remove organic contaminants from the NC film and improve the interface between neighboring NCs, facilitating charge transport.

\section{Ab initio simulations}


Ground-state electronic structure and geometry optimization for $\mathrm{ZnS}$ and $\mathrm{ZnO}$ was performed at the density functional level of theory using the Quantum ESPRESSO software package.56 All calculations employed the PBE exchange-correlation functional, ${ }^{57}$ a plane wave basis set with a kinetic energy cutoff of $28 \mathrm{Ry}$ (280 Ry) for the description of Kohn-Sham orbitals (charge density), and Ultrasoft pseudopotentials. ${ }^{58}$ Optical spectra were instead evaluated with Norm Conserving pseudopotentials with 100 Ry energy cutoff, using the "epsilon.x" code, also included in the Quantum ESPRESSO suite, which implements a single particle band-to-band expression of the Drude-Lorentz expression. The $3 \mathrm{~d}$ electrons of $\mathrm{Zn}$ have been explicitly included in the valence shell. Underestimation of the band gap with the standard GGA type functional was corrected by including a Hubbard-like potential on both $3 d$ orbitals of zinc $\left(\mathrm{U}_{\mathrm{Zn}}\right)$ and $2 \mathrm{p}$ orbitals of oxygen $\left(U_{0}\right)$ or sulfur $\left(U_{S}\right)$, along the lines described in the literature. ${ }^{59,60}$ The optimized values for the compounds $\left(\mathrm{U}_{\mathrm{Zn}}=12.0 \mathrm{eV}, \mathrm{U}_{0}=6.5 \mathrm{eV}\right.$ for $\mathrm{ZnO}$, and $\mathrm{U}_{\mathrm{Zn}}=13.75 \mathrm{eV}, \mathrm{U}_{\mathrm{S}}=$ $3.51 \mathrm{eV}$ for $\mathrm{ZnS}$ ) were obtained by means of the pseudohybrid Hubbard Density Functional approach (namely ACBN0), recently proposed by Agapito and coworkers. ${ }^{61}$ The effects of the ACBN0 approach on the structural, electronic and vibrational properties of $\mathrm{ZnO}$ and $\mathrm{ZnS}$, along with chalcogenides and metal oxides have been largely tested in previous reports. ${ }^{15,62,63}$

The (wurtzite) $\mathrm{ZnO}$ and (zincblende) $\mathrm{ZnS}$ bulk crystals and their derivatives obtained including substitutional In at Zn sites were simulated using periodic supercells, including 16/32 primitive cells respectively (i.e. 64 atoms). The Brillouin zone of the reciprocal lattice was sampled by using a uniform $(4 \times 4 \times 4)$ grid of k-points. All structures were relaxed until forces on all atoms were lower than $0.03 \mathrm{eV} \AA^{-1}$. In order to parallel the experimental result, and in view of the large In dosages involved in the experiment, we optimized the lattice parameters of the host (Figure S3 in ESI).

\section{Characterization techniques}

Optical absorption spectra of NCs dispersed in chloroform and of thin films deposited on quartz slides were obtained on an Agilent Cary60 UV-Vis spectrophotometer. Photoluminescence (PL) spectra were acquired with a Horiba Jobin Yvon Fluoromax-4 fluorometer with an excitation wavelength of $260 \mathrm{~nm}$. X-ray diffraction (XRD) patterns of dried NCs powders were collected using a Bruker D4 Endeavor diffractometer equipped with a $\mathrm{Cu}-\mathrm{K} \alpha$ radiation source and operated at $40 \mathrm{kV}$ 
and $35 \mathrm{~mA}$. The crystallite size was evaluated with the Scherrer relationship using the full width at half-maximum of the three main peaks fitted using Pseudo-Voigt functions. For each sample the extracted size values from each of the three diffraction peaks were averaged and the error was calculated as one standard deviation. Scanning electron microscopy (SEM) images were acquired on a FEI Verios 460L SEM operated at $3 \mathrm{kV}$ and 13 pA. Energy dispersive X-ray (EDX) spectra were acquired on a Nova 200 NanoSEM with a voltage of $15 \mathrm{kV}$. Transmission electron microscopy (TEM) images were acquired on a JEOL 2100F microscope operated at $80 \mathrm{kV}$. Raman spectra of samples deposited on silicon wafers were acquired on a Horiba LabRAM HR Evolution Raman spectrometer using a $532 \mathrm{~nm}$ laser at $12.5 \mathrm{~mW}$ power. The chemical composition of the samples was analyzed with atomic emission spectroscopy (AES) using an Agilent 4200 MP-AES instrument after digesting the solution in concentrated hydrochloric acid. Ellipsometric measurements were conducted on a J.A. Woollam M2000 spectroscopic ellipsometer at 75 deg incidence. The electrical measurements as a function of UV light illumination were conducted on $\mathrm{ZnS}$ thin films deposited onto pre-patterned electrodes (see SI for details on electrodes fabrication). The light and dark conductivity was measured using a Keysight 2900 sourcemeter applying a constant voltage of $0.5 \mathrm{~V}$. Light-emitting diodes (LED, Thorlabs, Inc.) of two different wavelengths (365 nm and $280 \mathrm{~nm}$ ) were utilized as sources for UVA and UVB/C radiation, respectively. The illumination power was calibrated using a commercial UV-enhanced silicon photodetector (Newport Corporation) and kept constant at $3 \mathrm{~mW} / \mathrm{cm}^{2}$.

\section{Conflict of interest}

There are no conflicts of interest to declare.

\section{Acknowledgements}

EDG and SW thank RMIT University for a Vice Chancellor Fellowship. The Australian Research Council is acknowledged for supporting this work through two Discovery Early Career Researcher Awards (DE170100164, EDG; DE150100427, JvE). The authors acknowledge the facilities and the technical assistance of the RMIT University's Microscopy and Microanalysis Facility (RMMF). 


\section{References}

1. L. E. Brus, The Journal of Chemical Physics, 1984, 80, 4403-4409.

2. C. B. Murray, D. J. Norris and M. G. Bawendi, Journal of the American Chemical Society, 1993, 115, 8706-8715.

3. K. S. Gu and H. Taeghwan, Small, 2011, 7, 2685-2702.

4. J. van Embden, A. S. R. Chesman and J. J. Jasieniak, Chemistry of Materials, 2015, 27, 2246-2285.

5. J. Hühn, C. Carrillo-Carrion, M. G. Soliman, C. Pfeiffer, D. Valdeperez, A. Masood,

I. Chakraborty, L. Zhu, M. Gallego, Z. Yue, M. Carril, N. Feliu, A. Escudero, A. M. Alkilany, B. Pelaz, P. del Pino and W. J. Parak, Chemistry of Materials, 2017, 29, 399-461.

6. J. Owen and L. Brus, Journal of the American Chemical Society, 2017, 139, 1093910943.

7. J. P. Wolfgang, G. Daniele, P. Teresa, Z. Daniela, M. Christine, C. W. Shara, B. Rosanne, A. L. G. Mark, A. L. Carolyn and A. P. Alivisatos, Nanotechnology, 2003, 14, R15.

8. M. V. Kovalenko, L. Manna, A. Cabot, Z. Hens, D. V. Talapin, C. R. Kagan, V. I. Klimov, A. L. Rogach, P. Reiss, D. J. Milliron, P. Guyot-Sionnnest, G. Konstantatos, W. J. Parak, T. Hyeon, B. A. Korgel, C. B. Murray and W. Heiss, ACS Nano, 2015, 9, 1012-1057.

9. D. V. Talapin, J.-S. Lee, M. V. Kovalenko and E. V. Shevchenko, Chemical Reviews, 2010, 110, 389-458.

10. I. J. Kramer and E. H. Sargent, ACS Nano, 2011, 5, 8506-8514.

11. R. Costi, A. E. Saunders and U. Banin, Angewandte Chemie International Edition, 2010, 49, 4878-4897.

12. Y. Gai, J. Li, B. Yao and J.-B. Xia, Journal of Applied Physics, 2009, 105, 113704.

13. B. B. Srivastava, S. Jana, N. S. Karan, S. Paria, N. R. Jana, D. D. Sarma and N. Pradhan, The Journal of Physical Chemistry Letters, 2010, 1, 1454-1458.

14. D. J. Norris, A. L. Efros and S. C. Erwin, Science, 2008, 319, 1776-1779.

15. P. D'Amico, A. Calzolari, A. Ruini and A. Catellani, Scientific Reports, 2017, 7, 16805.

16. S. Sapra, A. Prakash, A. Ghangrekar, N. Periasamy and D. D. Sarma, The Journal of Physical Chemistry B, 2005, 109, 1663-1668.

17. X. Fang, T. Zhai, U. K. Gautam, L. Li, L. Wu, Y. Bando and D. Golberg, Progress in Materials Science, 2011, 56, 175-287.

18. H. Althues, R. Palkovits, A. Rumplecker, P. Simon, W. Sigle, M. Bredol, U. Kynast and S. Kaskel, Chemistry of Materials, 2006, 18, 1068-1072.

19. J. van Embden, J. Jasieniak and P. Mulvaney, Journal of the American Chemical Society, 2009, 131, 14299-14309.

20. J. Joo, H. B. Na, T. Yu, J. H. Yu, Y. W. Kim, F. Wu, J. Z. Zhang and T. Hyeon, Journal of the American Chemical Society, 2003, 125, 11100-11105.

21. J. Zhang, B. Han, J. Liu, X. Zhang, Z. Liu and J. He, Chemical Communications, 2001, DOI: 10.1039/B109802K, 2724-2725.

22. J. W. Thomson, K. Nagashima, P. M. Macdonald and G. A. Ozin, Journal of the American Chemical Society, 2011, 133, 5036-5041.

23. K. Manzoor, S. R. Vadera, N. Kumar and T. R. N. Kutty, Applied Physics Letters, 2004, 84, 284-286.

24. A. Antonello, G. Brusatin, M. Guglielmi, A. Martucci, V. Bello, G. Mattei, P. Mazzoldi and G. Pellegrini, Thin Solid Films, 2010, 518, 6781-6786. 
25. B. Mashford, J. Baldauf, T.-L. Nguyen, A. M. Funston and P. Mulvaney, Journal of Applied Physics, 2011, 109, 094305.

26. W. G. Nilsen, Physical Review, 1969, 182, 838-850.

27. J. H. Kim, H. Rho, J. Kim, Y. J. Choi and J. G. Park, Journal of Raman Spectroscopy, 2012, 43, 906-910.

28. A. Antonello, G. Brusatin, M. Guglielmi, V. Bello, G. Mattei, G. Zacco and A. Martucci, Journal of Nanoparticle Research, 2011, 13, 1697-1708.

29. Y. Gao and P. Yin, Scientific Reports, 2017, 7, 43602.

30. Y. C. Cheng, C. Q. Jin, F. Gao, X. L. Wu, W. Zhong, S. H. Li and P. K. Chu, Journal of Applied Physics, 2009, 106, 123505.

31. Q. Xiong, J. Wang, O. Reese, L. C. Lew Yan Voon and P. C. Eklund, Nano Letters, 2004, 4, 1991-1996.

32. Notably, bulk values range between 3.5 and $3.8 \mathrm{eV}$ depending on the literature reference and on the crystalline structure of $\mathrm{ZnS}$, with the band gap of wurtzite being slightly larger than that of sphalerite.

33. M. P. Moloney, Y. K. Gun'ko and J. M. Kelly, Chemical Communications, 2007, DOI: 10.1039/B704636G, 3900-3902.

34. A. J. Morfa, G. Beane, B. Mashford, B. Singh, E. Della Gaspera, A. Martucci and P. Mulvaney, The Journal of Physical Chemistry C, 2010, 114, 19815-19821.

35. Y. Liu, H. Zhang, X. An, C. Gao, Z. Zhang, J. Zhou, M. Zhou and E. Xie, Journal of Alloys and Compounds, 2010, 506, 772-776.

36. X. D. Pi, R. Gresback, R. W. Liptak, S. A. Campbell and U. Kortshagen, Applied Physics Letters, 2008, 92, 123102.

37. D. Mocatta, G. Cohen, J. Schattner, O. Millo, E. Rabani and U. Banin, Science, 2011, 332, 77-81.

38. B. N., M. S., B. J.C., B. N. T. and B. S., physica status solidi (a), 2001, 184, 179-186.

39. I. Tsuji, H. Kato, H. Kobayashi and A. Kudo, Journal of the American Chemical Society, 2004, 126, 13406-13413.

40. L. A. Swafford, L. A. Weigand, M. J. Bowers, J. R. McBride, J. L. Rapaport, T. L. Watt, S. K. Dixit, L. C. Feldman and S. J. Rosenthal, Journal of the American Chemical Society, 2006, 128, 12299-12306.

41. E. Della Gaspera, A. S. R. Chesman, J. van Embden and J. J. Jasieniak, ACS Nano, $2014,8,9154-9163$.

42. S. Ghosh, M. Saha and S. K. De, Nanoscale, 2014, 6, 7039-7051.

43. E. Della Gaspera, M. Bersani, M. Cittadini, M. Guglielmi, D. Pagani, R. Noriega, S. Mehra, A. Salleo and A. Martucci, Journal of the American Chemical Society, 2013, 135, 3439-3448.

44. J. van Embden, A. S. R. Chesman, E. Della Gaspera, N. W. Duffy, S. E. Watkins and J. J. Jasieniak, Journal of the American Chemical Society, 2014, 136, 5237-5240.

45. A. S. R. Chesman, J. van Embden, E. Della Gaspera, N. W. Duffy, N. A. S. Webster and J. J. Jasieniak, Chemistry of Materials, 2014, 26, 5482-5491.

46. E. Della Gaspera, M. Bersani, G. Mattei, T.-L. Nguyen, P. Mulvaney and A. Martucci, Nanoscale, 2012, 4, 5972-5979.

47. F. Paglia, D. Vak, J. van Embden, A. S. R. Chesman, A. Martucci, J. J. Jasieniak and E. Della Gaspera, ACS Applied Materials \& Interfaces, 2015, 7, 25473-25478.

48. K. Nicholas, S. Birendra and M. Paul, Advanced Materials Interfaces, 2016, 3, 1600868 .

49. D.R. Lide, CRC Handbook of Chemistry and Physics, 87th edition, Taylor and Francis, Boca Raton, FL, 2007, 10-248

50. D. A. G. Bruggeman, Annalen der Physik, 1935, 416, 636-664. 
51. F. Todescato, A. S. R. Chesman, A. Martucci, R. Signorini and J. J. Jasieniak, Chemistry of Materials, 2012, 24, 2117-2126.

52. L. Larina, D. Shin, J. H. Kim and B. T. Ahn, Energy \& Environmental Science, 2011, 4, 3487-3493.

53. N. Nasiri, R. Bo, T. F. Hung, V. A. L. Roy, L. Fu and A. Tricoli, Advanced Functional Materials, 2016, 26, 7359-7366.

54. G. Philipp, W. Sumeet, S. Sharath and B. Madhu, Advanced Electronic Materials, $2015,1,1500264$.

55. Only highly quantum confined $\mathrm{ZnO}$ clusters have a large enough band gap to be fully transparent to UVA radiation, but they are very unstable and tend to grow, with the consequent reduction in band gap energy into the UVA spectral region.

56. G. Paolo, B. Stefano, B. Nicola, C. Matteo, C. Roberto, C. Carlo, C. Davide, L. C. Guido, C. Matteo, D. Ismaila, C. Andrea Dal, G. Stefano de, F. Stefano, F. Guido, G. Ralph, G. Uwe, G. Christos, K. Anton, L. Michele, M.-S. Layla, M. Nicola, M. Francesco, M. Riccardo, P. Stefano, P. Alfredo, P. Lorenzo, S. Carlo, S. Sandro, S. Gabriele, P. S. Ari, S. Alexander, U. Paolo and M. W. Renata, Journal of Physics: Condensed Matter, 2009, 21, 395502.

57. J. P. Perdew, K. Burke and M. Ernzerhof, Physical Review Letters, 1996, 77, 3865 3868.

58. D. Vanderbilt, Physical Review B, 1990, 41, 7892-7895.

59. M. Korotin, T. Fujiwara and V. Anisimov, Physical Review B, 2000, 62, 5696-5699.

60. S.-G. Park, B. Magyari-Köpe and Y. Nishi, Physical Review B, 2010, 82, 115109.

61. L. A. Agapito, S. Curtarolo and M. Buongiorno Nardelli, Physical Review X, 2015, 5, 011006.

62. A. Catellani, A. Ruini and A. Calzolari, Journal of Materials Chemistry C, 2015, 3, 8419-8424.

63. P. Gopal, M. Fornari, S. Curtarolo, L. A. Agapito, L. S. I. Liyanage and M. B. Nardelli, Physical Review B, 2015, 91, 245202. 